\title{
Recent results on nucleon sigma terms in lattice QCD
}

\author{
R. D. Young and A. W. Thomas ${ }^{\mathrm{b}}$

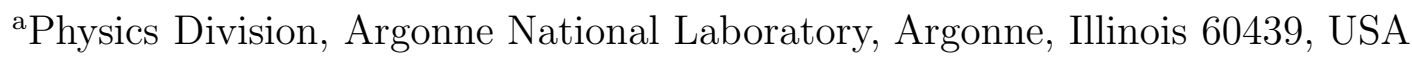 \\ ${ }^{\mathrm{b}} \mathrm{CSSM}$, School of Chemistry and Physics, University of Adelaide, Adelaide, South \\ Australia 5005, Australia
}

It has proven a significant challenge to experiment and phenomenology to extract precise values of the nucleon sigma terms. This difficulty opens the window for lattice QCD simulations to lead the field in resolving this aspect of nucleon structure. Here we report on recent advances in the extraction of nucleon sigma terms in lattice QCD. In particular, the strangeness component is now being resolved to a precision that far surpasses best phenomenological estimates.

\section{NUCLEON SIGMA TERMS}

The nucleon sigma terms are important quantities in resolving the dynamics of QCD, where they help to understand the role of explicit chiral symmetry breaking in the mass of the nucleon. Indeed, the strangeness component plays a unique role in that is purely a vacuum polarization effect, analogous to the Lamb shift in QED. Further, the strange quark is light enough that it probes the nonperturbative distance scales of QCD. Beyond the fundamental importance to nucleon structure, the strange quark condensate in the nucleon is of significant interest in studies of the QCD phase structure at large baryon density [1] and in constraining predicted cross sections for dark matter detection [2]3].

The sigma terms of the nucleon are defined by the scalar form factors in the limit of vanishing momentum transfer, $\sigma_{q}=m_{q}\langle N|\bar{q} q| N\rangle$, where $q$ denotes the quark flavour of interest. The light-quark sigma term (or pion-nucleon sigma term), $\sigma_{\ell}=m_{\ell}\langle\bar{u} u+\bar{d} d\rangle$ (with $m_{\ell} \equiv\left(m_{u}+m_{d}\right) / 2$ ), is related to $\pi-N$ scattering through a chiral low-energy relation [4,5]6],

$\Sigma_{\pi N} \equiv \sigma_{\ell}=\Sigma_{\pi N}^{\mathrm{CD}}-\Delta_{R}-\Delta_{\sigma}$,

where the experimental input required is the Born-subtracted, isoscalar $\pi N$ scattering amplitude evaluated at the (unphysical) Cheng-Dashen point, $\Sigma_{\pi N}^{\mathrm{CD}}$. The smallness of the up and down quark masses ensures that both the remainder term, $\Delta_{R}$ [5:7], and the form factor correction, $\Delta_{\sigma}[8]$, can be reliably estimated. An early analysis of experimental results [9] led to the value $\sigma_{\ell}=45 \pm 8 \mathrm{MeV}$ [8], while a more recent analysis suggested a higher value, $64 \pm 7 \mathrm{MeV}[10]$.

Unlike the light quarks, the strange quark is too heavy to invoke a low-energy relation. Therefore $\sigma_{s}$ has generally been estimated by studying the breaking of $\mathrm{SU}(3)$ within the 
baryon octet [1,11/2]. Here, the observed spectrum has been used to derive a constraint on the non-singlet combination

$\sigma_{0}=m_{\ell}\langle N|\bar{u} u+\bar{d} d-2 \bar{s} s| N\rangle$,

where chiral effective field theory studies lead to a value $\sigma_{0}=36 \pm 7 \mathrm{MeV}$ [12], building on earlier estimates by Gasser [11]. The difference between $\sigma_{0}$ and the extracted $\sigma_{\ell}$ then defines the strange-quark contribution, $\sigma_{\ell}-\sigma_{0}=2 \frac{m_{\ell}}{m_{s}} \sigma_{s}$. Equivalently, the relative strangeness component is often discussed in terms of the parameter $y$,

$\frac{\sigma_{0}}{\sigma_{\ell}}=1-\frac{2\langle N|\bar{s} s| N\rangle}{\langle N|\bar{u} u+\bar{d} d| N\rangle} \equiv 1-y$.

Taking the above pion-nucleon sigma term values and the estimated $\sigma_{0}$ gives values $y \simeq$ $0.2 \pm 0.2$ and $0.44 \pm 0.13$, and using a strange-to-light quark mass ratio of $\sim 25$ gives strangeness sigma terms, $\sigma_{s}=110 \pm 130$ and $350 \pm 120 \mathrm{MeV}$, respectively. This approach clearly leads to a result for $\sigma_{s}$ which is very sensitive to the precise value of $\sigma_{\ell}$. We also note that even with perfect $\pi-N$ data to better constrain $\sigma_{\ell}$, the $7 \mathrm{MeV}$ uncertainty in $\sigma_{0}$ alone leads to $\mathrm{a} \sim 90 \mathrm{MeV}$ uncertainty in $\sigma_{s}$. This limitation clearly opens the way for lattice QCD to offer significant improvement.

\section{LATTICE CALCULATIONS}

\subsection{Light quarks}

In early lattice simulations, limited computing power forced the use of heavy quarks and the quenching of the light quarks in the theory [13,14]. The first direct calculations of the disconnected contributions (though still on a quenched gauge ensemble), including an estimate of the strange quark contributions, were performed in the work of Fukugita et al. [15] and Dong et al. [16]. In these works, the $\pi-\mathrm{N}$ sigma was found to be consistent with observation - see Figure 1 for a summary of results.

The first determination using (2 flavours of) dynamical quarks found a value for $\sigma_{\ell}$ that is smaller than phenomenological expectations [17]. A notable feature of this calculation is that consistent results were demonstrated between the direct calculation of the disconnected loops and that extracted from the fit to the nucleon mass, using the Feynman-Hellman relation 1

$\sigma_{q}=m_{q} \frac{\partial M_{N}}{\partial m_{q}}$

While this calculation appeared to yield a value that was rather low, it was soon identified that the sigma term is particularly sensitive to chiral extrapolation [19]. After this time, most extractions of $\sigma_{\ell}$ have come by application of the Feynman-Hellman relation to the mass extrapolation [20,21,22,23,24,25,26].

It was common in the early calculations that the quoted error only reflected the statistical uncertainty in the underlying extraction. The work of Procura et al. [22] made a detailed investigation of the systematic errors associated with the input parameters of

\footnotetext{
${ }^{1}$ Commonly the quark masses in this relation are replaced by the meson mass squared using the GellMann-Oakes-Renner relation.
} 


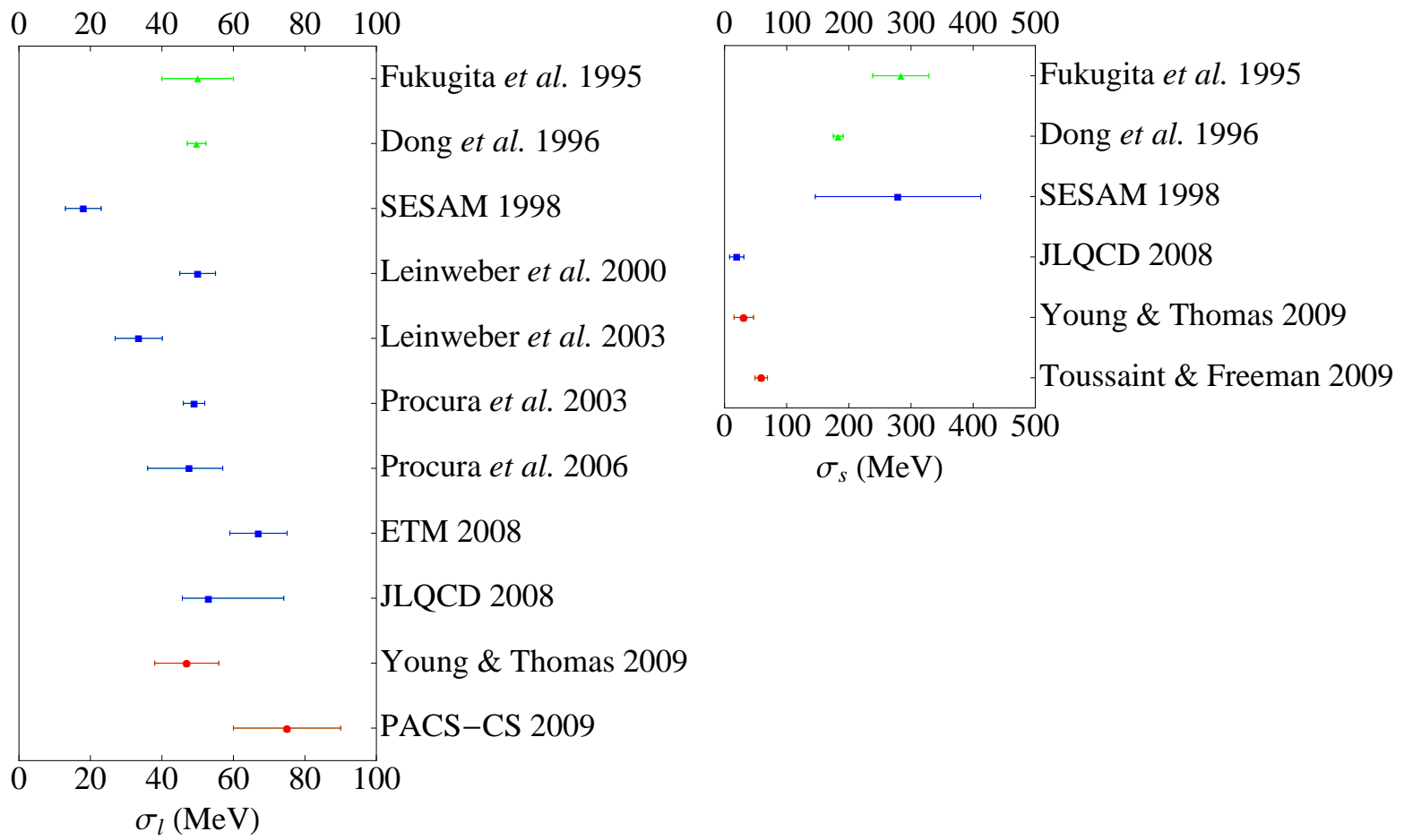

Figure 1. Summary of lattice QCD calculations of the light-quark sigma term (left panel) and strangeness sigma term (right panel). Triangles, squares and circles denote quenched, 2 -flavour and 2+1-flavour lattices, respectively. We caution that different calculations have invested differing degrees of effort into quantifying their error estimate.

their functional form. Contrasting this with their earlier work [21], where only the statistical error was quoted, is a useful guide as to the potential size of systematic effects. Of course, various systematics will continue to be better controlled as the simulation results improve.

While it is not obvious from Figure1(a) that the lattice results are showing convergence - without taking bias - there does appear to be consensus that the lattice results are consistent with the two phenomenological extractions discussed above.

\subsection{Strange quark}

Early lattice estimates of the strangeness sigma term supported the rather large values that had been inferred from phenomenology [15,16]. Going beyond the quenched approximation, the 2-flavour dynamical calculations of the SESAM Collaboration [17] also supported the larger strangeness values, though with substantial uncertainty. Another early calculation highlighted some of the challenges of resolving these matrix elements and concluded that the strangeness component is most likely rather small [18].

While the strange quark in the 2-flavour simulations is essentially quenched, it is interesting to consider the light-quark disconnected diagrams, which are truly dynamical. One can consider the ratio of the disconnected to connected (or sea to valence) contributions 
to the sigma term,

$R_{d / c}=\frac{\langle N|\bar{u} u+\bar{d} d| N\rangle_{d i s c}}{\langle N|\bar{u} u+\bar{d} d| N\rangle_{c o n}}$

In the quenched simulations a relatively large value for this ratio was observed, with $R_{d / c}=2.23(52)$ [15] and 1.79(7) [16]. We note that the effect of unqenching appears to reduce this ratio, with the corrsponding ratio found to be $R_{d / c}=1.26(57)$ in the dynamical simulation [17]. This indicates a decrease in the relative strength of the disconnected component.

Extending this discussion, a modern determination of this ratio by JLQCD [24] has found $R_{d / c}=0.41(5)$ (taking PQ-b as the "best estimate" ) - suggesting even further reduction of this ratio as results better describe the physical regime. This study constrained the nucleon mass using light-quark masses taking values over the range $m_{s} / 6-m_{s}$ (or pion masses $\sim 290-750 \mathrm{MeV})$.

In a SU(3) symmetric $\left(m_{u}=m_{d}=m_{s}\right)$ 3-flavour dynamical calculation, the disconnected contributions of each of the three quarks are identical, satisfying the identity

$2\langle N|\bar{s} s| N\rangle_{\text {disc }} \stackrel{S U(3)}{=}\langle N|\bar{u} u+\bar{d} d| N\rangle_{\text {disc }}$.

In a 2-flavour simulation, the literal $\bar{s} s$ contribution is zero, yet it could be reasonable to adopt the above relation to infer the $\bar{s} s$ from a 2 -flavour calculation. Indeed this is the approach taken by [24], where at the SU(3) symmetric point one can infer $y \sim 0.09$. Further taking the 2-flavour result for the increase in both the connected and disconnected light-quark terms as the light-quarks are reduced to the physical point reduces the estimate of $y$ to $\sim 0.03[24$ ] (assuming that the strangeness term is only weakly dependent on the light-quark masses). Indeed, when combined with their extraction for the $\sigma_{\ell}$, this leads to a significantly reduced value of the strangeness sigma term $\sigma_{s}=20 \pm 12 \mathrm{MeV}$.

The major cause of this reduction compared with the previous estimates comes from the observation that the derivative of the nucleon mass $\left(\frac{\partial M_{N}}{\partial m_{s e a}}\right)$ is quite strongly dependent on the sea-quark mass $\left(m_{\text {sea }}\right)$. Indeed, this derivative was observed to be enhanced by a factor of $\sim 10$ in going from the strange-quark mass to the light-quark mass. Beyond the numerical improvements in the underlying lattice simulations, this study has also incorporated the consequences of chiral symmetry breaking, where there are known to be substantial chiral logarithms in these derivatives - leading to the observed enhancement in the light-quark domain.

These authors have recently reported results on testing their method against a direct calculation of the disconnected strangeness component, finding compatible results [27]. Further, the analysis of the JLQCD Collaboration is being extended to $2+1$ flavours of dynamical quarks, where preliminary results also support their 2-flavour findings [28].

Another opportunity to study the strange-quark mass dependence came with a series of calculations of the baryon spectrum in 2+1-flavour dynamical simulations which emerged in 2008 [29 30,31,32] (after an early venture in 2001 [33]). These new 3-flavour results allowed tests of the $\mathrm{SU}(3)$ chiral expansion at the lattice quark masses [29,25,26]. Indeed the poor convergence of the SU(3) expansion, already recognised at the physical quark masses [12,11, was rediscovered on the lattice. These three chiral-lattice papers took three different approaches to deal with this problem. PACS-CS [26] simply abondoned 
the SU(3) formulation; LHPC [29] sacrificed the coefficients of the chiral logarithms in order to stabilise the fits; we 25] introduced a single new fit parameter that acts to separate the low- and high-energy contributions to the chiral loop integrals.

The results of Ref. [25] built on the established benefits of using finite-range regularisation, which has previously been shown to dramatically improve the SU(3) convergence [11,34 and offer robust chiral extrapolation for lattice QCD [20,35]. The analysis of Ref. 25] demonstrated the ability to extrapolate in both the light- and strange-quark masses to accurately reproduce the physical octet baryon spectrum, and also extrapolate to the heavier quark masses (not used in the fits) as calculated on the lattice. The results were found to be consistent with two different lattice discretizations, which also allowed an estimate of potential discretization effects.

Using the Feynman-Hellman relation, we found $\sigma_{\ell}$ to be compatible with phenomenological and lattice estimates. And the strangeness sigma term was identified to be relatively small, supporting the 2-flavour calculation of JLQCD [24].

Another recent 2+1-flavour dynamical result for $\sigma_{s}$ has been reported by Toussaint and Freeman [36], using an application of the Feynman-Hellman relation on the nucleon correlator. This technique substantially differs from the approaches based on fitting the the nucleon mass and therefore provides a strong independent test. The result of Toussaint and Freeman [36] is marginally higher than the values reported by JLQCD [24] and by us [25]. Nevertheless, as seen in Figure 1(b), the modern lattice results for $\sigma_{s}$ are in agreement that the size of the strangeness sigma term is substantially smaller than has been previously thought.

\section{IMPACT}

As described above, the dominant uncertainty in knowledge of the scalar quark couplings has lies in the strangeness component. The current generation of lattice QCD calculations has primarily resolved this limitation by providing stringent new limits on the strange quark sigma term. As a result of this improvement, the predicted cross sections for models of dark matter can now be revised. In a recent paper, Giedt et al. [37] have investigated the impact of the new lattice QCD results on the cross sections for a class of supersymmetric models. The poorly known strangeness $\sigma$-term had previously resulted in cross sections which varied by roughly an order of magnitude [3]. The uncertainties have been dramatically reduced by the lattice calculations and importantly, one now has significant discrimination power between the ensemble of models considered.

This work was supported by the U.S. Department of Energy, Office of Nuclear Physics, under Contract No. DE-AC02-06CH11357; and the Australian Research Council.

\section{REFERENCES}

1. A. E. Nelson and D. B. Kaplan, Phys. Lett. B 192, 193 (1987).

2. A. Bottino et al., Astropart. Phys. 13, 215 (2000) arXiv:hep-ph/9909228]; Phys. Rev.

D 78, 083520 (2008) arXiv:0806.4099 [hep-ph]].

3. J. R. Ellis et al., Phys. Rev. D 77, 065026 (2008) [arXiv:0801.3656 [hep-ph]].

4. T. P. Cheng and R. F. Dashen, Phys. Rev. Lett. 26, 594 (1971).

5. L. S. Brown, W. J. Pardee and R. D. Peccei, Phys. Rev. D 4, 2801 (1971). 
6. J. Gasser, H. Leutwyler and M. E. Sainio, Phys. Lett. B 253, 252 (1991).

7. V. Bernard et al., Phys. Lett. B 389, 144 (1996) arXiv:hep-ph/9607245.

8. J. Gasser, H. Leutwyler and M. E. Sainio, Phys. Lett. B 253, 260 (1991).

9. R. Koch, Z. Phys. C 15, 161 (1982).

10. M. M. Pavan et al., PiN Newslett. 16, 110 (2002) |arXiv:hep-ph/0111066].

11. J. Gasser, Annals Phys. 136, 62 (1981).

12. B. Borasoy and U.-G. Meißner, Annals Phys. 254, 192 (1997) arXiv:hep-ph/9607432.

13. L. Maiani et al., Nucl. Phys. B 293, 420 (1987).

14. S. Güsken et al. [SESAM], Phys. Lett. B 212, 216 (1988).

15. M. Fukugita et al., Phys. Rev. D 51, 5319 (1995) |arXiv:hep-lat/9408002].

16. S. J. Dong et al., Phys. Rev. D 54, 5496 (1996) arXiv:hep-ph/9602259].

17. S. Güsken et al. [SESAM], Phys. Rev. D 59, 054504 (1999) [arXiv:hep-lat/9809066].

18. C. Michael et al. [UKQCD], Nucl. Phys. Proc. Suppl. 106, 293 (2002) arXiv:hep-lat/0109028].

19. D. B. Leinweber et el., Phys. Lett. B 482, 109 (2000) [arXiv:hep-lat/0001007].

20. D. B. Leinweber et al., Phys. Rev. Lett. 92, 242002 (2004) [arXiv:hep-lat/0302020].

21. M. Procura et al., Phys. Rev. D 69, 034505 (2004) [arXiv:hep-lat/0309020].

22. M. Procura et al., Phys. Rev. D 73, 114510 (2006) arXiv:hep-lat/0603001.

23. C. Alexandrou et al. [ETM], Phys. Rev. D 78, 014509 (2008) [arXiv:0803.3190 [heplat]].

24. H. Ohki et al. [JLQCD], Phys. Rev. D 78, 054502 (2008) arXiv:0806.4744 [hep-lat]].

25. R. D. Young and A. W. Thomas, arXiv:0901.3310 [hep-lat].

26. K. I. Ishikawa et al. [PACS-CS], Phys. Rev. D 80, 054502 (2009) arXiv:0905.0962 [hep-lat]].

27. K. Takeda et al. [JLQCD], arXiv:0910.5036 [hep-lat].

28. H. Ohki et al. [JLQCD], arXiv:0910.3271 [hep-lat].

29. A. Walker-Loud et al. [LHPC], Phys. Rev. D 79, 054502 (2009) arXiv:0806.4549 [hep-lat]].

30. S. Aoki et al. [PACS-CS], Phys. Rev. D 79, 034503 (2009) arXiv:0807.1661 [hep-lat]]. 31. H. W. Lin et al. [HSC], Phys. Rev. D 79, 034502 (2009) [arXiv:0810.3588 [hep-lat]].

32. S. Dürr et al., Science 322, 1224 (2008) arXiv:0906.3599 [hep-lat]].

33. C. W. Bernard et al., Phys. Rev. D 64, 054506 (2001) arXiv:hep-lat/0104002.

34. J. F. Donoghue et al., Phys. Rev. D 59, 036002 (1999) [arXiv:hep-ph/9804281].

35. D. B. Leinweber et al., Phys. Rev. D 61, 074502 (2000) |arXiv:hep-lat/9906027).

36. D. Toussaint and W. Freeman [MILC], Phys. Rev. Lett. 103, 122002 (2009) arXiv:0905.2432 [hep-lat]].

37. J. Giedt et al., to appear Phys. Rev. Lett., arXiv:0907.4177 [hep-ph]. 